\title{
New demands on inspection planning and quality testing for micro- and nanostructured components
}

\author{
Karina Weißensee \\ Department of Quality Assurance and Industrial Image Processing, Ilmenau University of Technology, PB 100565, 98684 IImenau, Germany
}

ABSTRACT

The development and control of more and more complex and extensive technical systems yields to measurement-technology requirements in an increasing degree. These requirements can not be met with the exclusive operation mode of a single-sensor measuring instrument because in most instances multifarious and multistructured measuring quantities are existing on one device under test. The aim of this paper is to discuss new problems of inspection planning arising from the improvement in measurement technology. Essential demands, ideas and conceptual approaches to multistructured quality inspections will be presented.

Keywords: Inspection planning; measuring strategy; micro- and nano metrology

Citation: Karina Weißensee, "New demands on inspection planning and quality testing for micro- and nanostructured components", Acta IMEKO, vol. 2, no. 1, article 15, August 2013, identifier: IMEKO-ACTA-02(2013)-01-15

Editors: Paolo Carbone, University of Perugia, Italy; Gerhard Linß, Ilmenau University of Technology, Germany

Received March 27 ${ }^{\text {th }}, 2013$; In final form April 4 ${ }^{\text {th }}, 2013$; Published August 2013

Copyright: (C) 2013 IMEKO. This is an open-access article distributed under the terms of the Creative Commons Attribution 3.0 License, which permits unrestricted use, distribution, and reproduction in any medium, provided the original author and source are credited

Funding: The presented work is the result of the close interdisciplinary research within the SFB 622, which is located at the TU IImenau, Germany, and funded by the German Research Foundation (DFG)

Corresponding author: Karina Weissensee, e-mail: karina.weissensee@tu-ilmenau.de

\section{INTRODUCTION}

The dimensional characterisation of structures on industrial products is fundamental for the assessment of the functionality and for the quality control. Measuring tasks on micro- and nanostructured components are very complex and different due to their high variety on raw materials, functions and geometries. The industrial production of micro- and nanostructured parts and systems expedites the development of adequate metrology for quality control. Furthermore there is the industrial request for measuring instruments solving all measuring tasks on the measuring object preferably fully automated. This means all measuring tasks should be solved using only one instrument. Multisensor coordinate measuring machines are the metrological basis for using more sensors to solve various measuring tasks on one measuring object without fixing changes. These machines offer considerable advantages e. g. high flexibility, short measuring times and ways. But for an adequate usage there is the requirement of a high knowledgelevel on the parts of the user [1]. A first way to improve this situation is to develop tools which give assistance in inspection planning to the user.

In general, inspections of micro- and nanostructured components have to cope with a huge number of inspection features on one measuring object only. Typically very small features for example $100 \mathrm{~nm}$ wide structures are distributed over a large area of several square millimetres. This is a challenging task. With the nanopositioning and nanomeasuring machine developed at Ilmenau University of Technology, subnanometre resolution and nanometre uncertainty in a measuring volume of $25 \times 25 \times 5 \mathrm{~mm}^{3}$ have been demonstrated in the last few years. In practice, however, there are too many different requirements for sensing surfaces or for detecting structures [2]. In order to perform 3D-coordinate measurements within the micro- and nanometre range a combination of different sensors must be utilised [3]. These sensors should be different in relation to their physical measuring principle and their measuring scale.

There are several physical principles according to which probes can be realized. Every probe, either non-tactile or tactile, shows specific advantages and disadvantages. Solving all these various measuring tasks is not possible by means of only optical or only tactile probes. It is very important to be able to select the optimal sensor for a specific measurement task [2].

In order to enable the automatic execution of inspection plans for micro- and nanostructured components the measuring machine must include a cascaded multisensor system. A cascaded multisensor system consists of multiple probing 


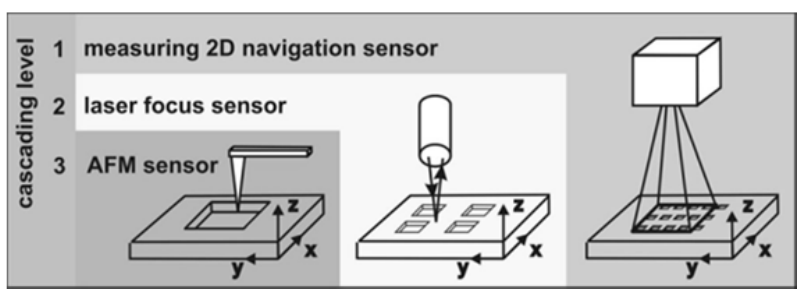

Figure 1. Setup of a cascaded multisensor system [3].

sensors with very different measuring range and very different measuring resolution (Figure 1). It is characterised by the internal information processing between the different sensors and enables the stage to stage accuracy-dependent inspection of micro- and nanostructured 3D features. Those specifics must be taken into account at inspection planning and at the execution of inspection plans [3].

In section 2 the main problems of inspection planning in the micro- and nanoscale will be summarised. Furthermore general tasks of inspection planning will be described. The topic of section 3 is the conceptual approach to an assistance tool for inspection planning. In section 4 possible concepts rules for measuring strategies will be discussed. Finally in the concluding section the major results are summarised.

\section{STATEMENT OF CURRENT PROBLEMS}

The main tasks of inspection planning can be presented as answers of the questions illustrated in Figure 2. The question: "What should be inspected?", yields to the measuring object and its inspection features. The question: "Who is to be responsible for the inspection?, or "Who should realise the measurement?", depends on the used measuring instruments and yields to the operators. "With which instrument should the inspection be executed?", yields to the measuring instruments especially adequate sensors. "How should the measurement be executed?", yields to the measuring strategy and is one of the most complicated questions. And finally "When should the inspection be executed?", yields to the course of action in the production chain and depends on the measuring time.

Furthermore there are interdependencies between the most parts of inspection planning. For example the measuring time depends on the measuring strategy and the selection of an adequate measuring instrument depends on the given tolerances and specifications of the inspection feature. The determination of a potential inspection strategy poses a big challenge for the inspection planner, especially if the sensor selection and the inspection strategy are correlated. All these elements of

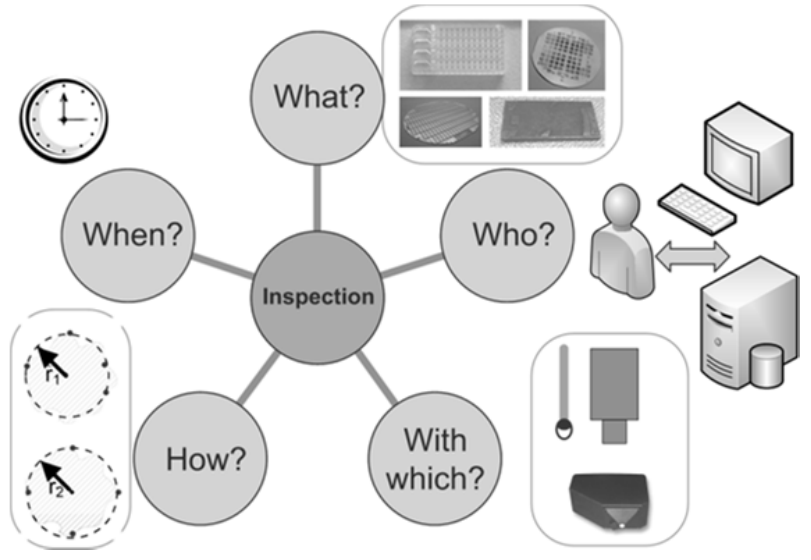

Figure 2. General tasks of inspection planning. inspection planning can not be established without considering all the others. Therefore often control loops are used for inspection planning [3].

Figure 3 illustrates the typical course of action of the planning process in a simplified way. The backward arrow represents the iterative process of sensor selection- and strategy planning based on the given tolerances of the inspection feature. Due to the large number of inspection features on multi-scaled devices under test, the realisation of the sensor selection in a manual way is very costly in terms of time, is based on the inspection planners know-how and can cause rough errors. The selection and assignment of capable sensors to the different inspection features of a micro- and nanostructured device under test is actually realised manually by the inspection planner. The inspection planner has to be well schooled in the technical characteristics of all available measuring instruments/sensors.

For measurements on micro- and nanostructured components different approaches are established. But not only the geometry of the measuring feature determines the sensor selection. Also structures in the work environment, which influence the accessibility, as well as material- and surface properties are crucial for the sensor selection [5]. Furthermore, the bandwidth of possible application areas is not exhaustively investigated yet for some of the newer sensors. For simplifying the process of inspection planning and to reduce quality cost, it should be worked out if the selection of a capable sensor can be

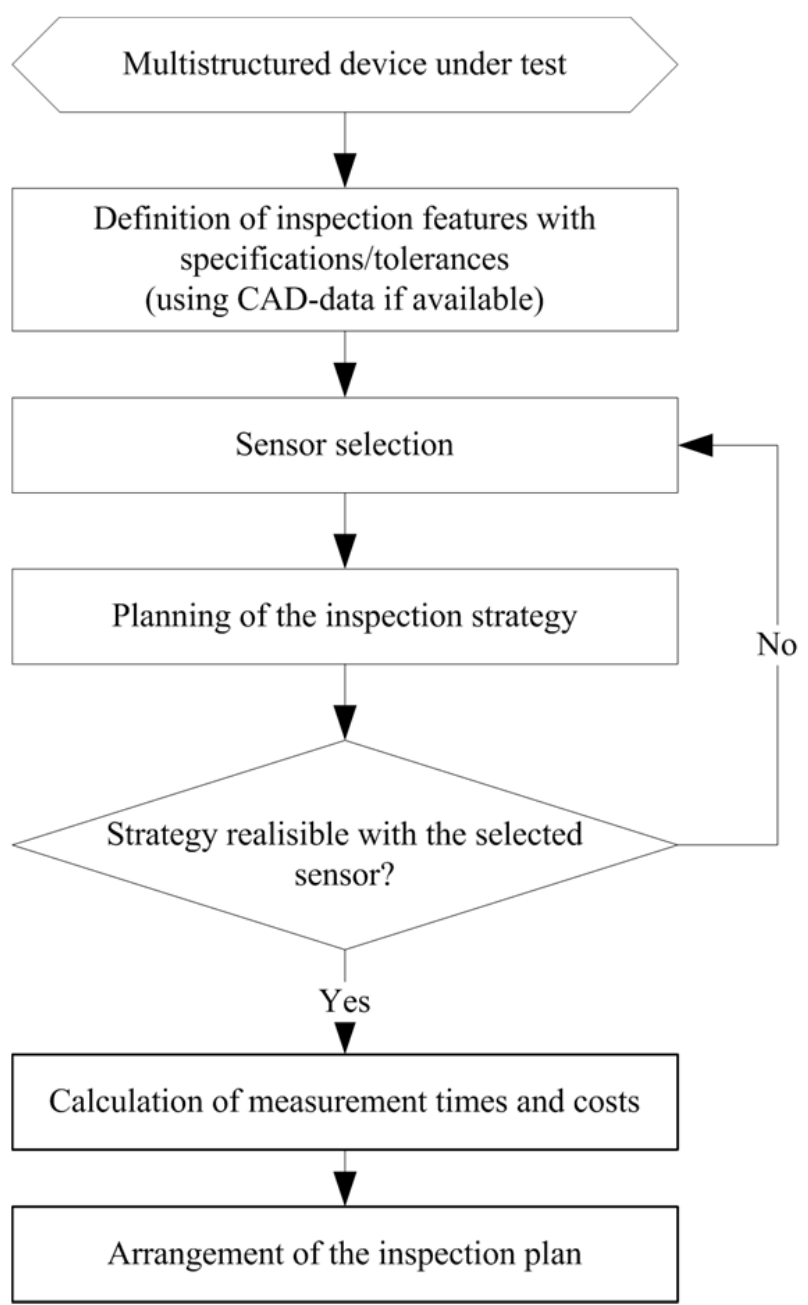

Figure 3. Typical course of action for inspection planning [4] 
realised automatically. In a first step an assistance tool for sensor selection should be prepared to check the user's acceptance, too [6]. Secondly, concepts for measuring strategies will be developed. Additionally in consequence of the large number of inspection features in the micro- and nanoscale huge measurement data will be generated. This requires adequate hard- and software systems for the data handling.

\section{ASSISTANCE TOOL FOR SENSOR SELECTION}

The main parts of inspection planning are especially the selection of a capable measuring instrument respectively sensor and the design of adequate inspection strategies. Therefore, extensive interrelations between the sensors and possible inspection features and also detailed information on sensor parameterisation have to be implemented. For general measurements the automated sensor selection would require an immense database with regularities for the assignment of a capable sensor and for the sensor parameterisation. Only for reiterative measurements, as usual in series production, the fully automated inspection planning is efficient and conceivable within custom-made measuring systems [4].

In a first step the methodology of sensor selection should be fragmented to prepare an assistance tool for inspection planning. From these items general rules should be deduced. The previous knowledge of sensors as well as materials and surfaces of devices under test should be provided by the assistance tool. If all information on available sensors is implemented, the sensor selection is a complex decision problem. In [7] an assistant system is presented which is divided into a hardware and a software system. The hardware assistant system uses general rules of thumb and expert knowledge whereby the software assistant system is based on simulations and uses neural networks and generic algorithms for the optimisation of the measurement.

\subsection{Acquisition of sensor-specific properties}

In dependence on the functional principle of the used sensors all for the inspection task relevant sensor-specific information should be determined and systematically classified. Therefore the inspection task based measurement uncertainty should be analysed for the determination of operation area limits. Operation areas of several sensors should be confined in relation to their application. Assets and drawbacks of several sensors should be experimentally analysed by means of selected samples of inspection features.

The technological prerequisites of measurements on microand nanostructured quality features have to be defined. Therefore material properties of the surface, technological manufacturing processes and the geometry of the inspection feature have to be taken into account. Additional intrinsic properties of the sensor have to be determined for the selection of an adequate inspection equipment respectively sensor. Within a database criteria for possible inspection features should be assigned to available sensors considering the specifications of the inspection feature. This database is the foundation of the automated sensor selection of micro- and nanostructured inspection features. Often a main part of necessary information on the inspection feature can be taken from the CAD-data. The sensor selection can be realised using an inference method based on the information about the measuring spectrum of each sensor stored in the database. Compulsory selection criteria are in addition to the technical feasibility, the measurement range of the available sensors and the maximum permissible measurement uncertainty corresponding to the specification of the quality feature. The sensor selection should be realised comprehensible and reproducible according to logical defined rules.

\subsection{Knowledge-based method}

Knowledge-based principles of decision theory are used for assigning inspection features and capable sensors. Thereby constructive algorithms should be used for the concentration of information and for the realisation of automation. It is the aim to reduce the influence of the inspection planner insofar as equal measuring tasks yield to the same sensor selection.

At the moment inspection planning is effected in dependence on the state of the planner's knowledge. For this reason it is not enough to upgrade the accuracy of the sensors. A multitude of measurement-specific parameters has to be determined for predefining the measuring strategy, which is an essential part of inspection planning. The parameter setting has a considerable effect on the measuring result. On that account it is needed to develop approaches to inspection planning in which the preparation of the inspection plan is less dependent on the individual measurement technician's state of knowledge.

The sensor selection takes up an essential position within the knowledge-based inspection planning. The automation of sensor selection would reduce the measurement times, the measurement uncertainty and would advance the usability as well as reduce the planning effort. Experiments to the measurability of different multistructured inspection features are realised as a first step to the central aim. In the following the stepwise procedural method of automated sensor selection using measurability experiments is itemised:

a) definition, characterisation and classification of the inspection feature

b) experiments to measurability on the available sensors/sensor systems

c) evaluation of experimental results:

- feasibility of the measuring task

- documentation of capable parameter settings and inspection strategies

- measuring condition requirements

- expression of the measurement uncertainty

- measuring time

d) transfer of results in the database.

Based on the measurement experiments a systematic structure has been developed for automated sensor selection, which depends on possible criteria e.g. physical measuring principle, dimensionality of the inspection structure (direction sensitivity) and surface properties.

Figure 4 demonstrates one of the experiments, especially the measurement of a microlens. The multi-scaled approach becomes apparent. Some inspection features especially the surface structure (Figure 4d)) are measurable only with highresolution sensors. The external dimensions of the lens are measurable with standard image sensors, whereas special properties, for example the edge (Figure $4 \mathrm{~b}$ ) and c)) are analysable only with high-resolution 3D-sensors.

A software tool with the implemented systematic structure for sensor selection has been developed for the inspection 


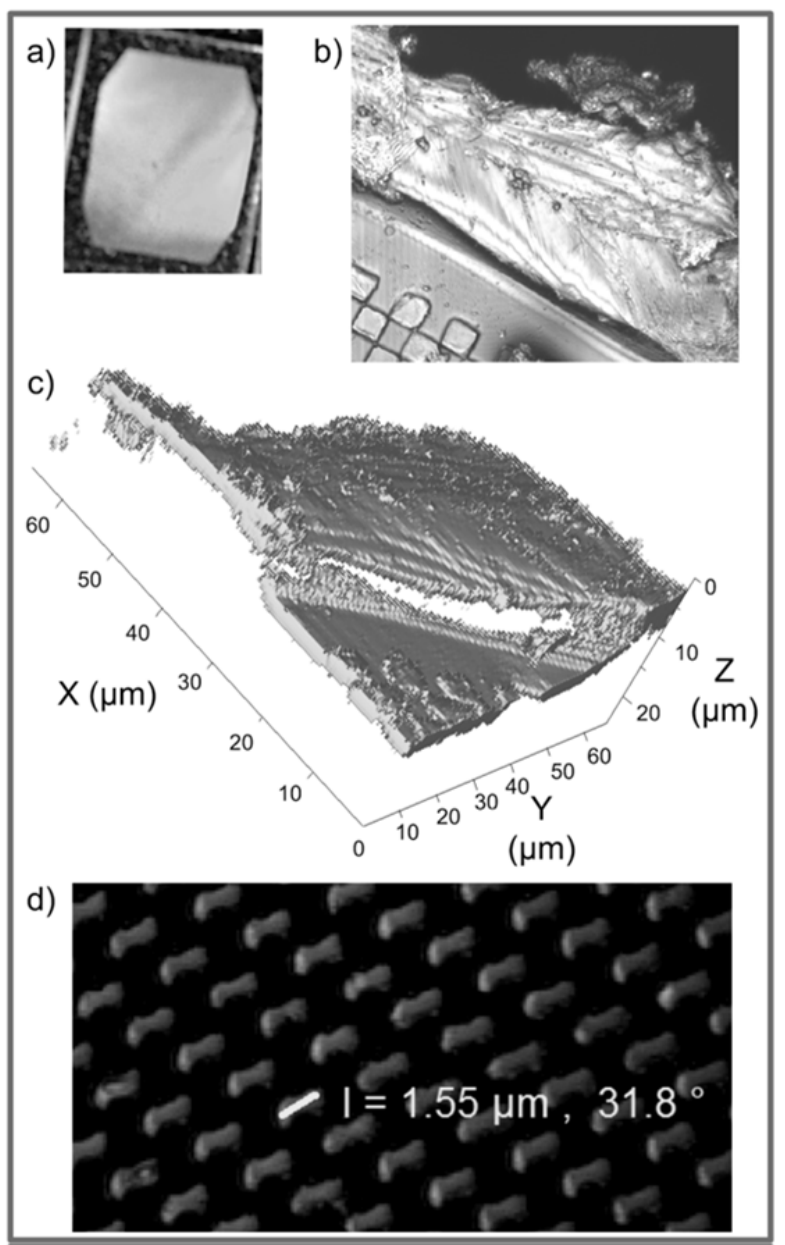

Figure 4. Examples of measurements on a micro lens; a) illustration of the lens (length $=9 \mathrm{~mm}$ ); b) topography view of an edge on the lens; c) 3D-view of the edge; d) surface measurement with laser scanning microscope [4].

planning support. The systematic of the software tool was verified based on test measurements.

The software tool distinguishes between typical devices under test and non-established inspection features. For typical or rather established mechanical components e.g. silicon wafers, ball-grid arrays or injectors varied experiences for capable measuring strategies are available. For this, current literature researches into new measuring methods and measuring results were executed and experts were consulted.

After selecting an established device under test, in the most cases, further properties for selection are offered. For nonestablished inspection features a systematic goal-oriented questioning-system is implemented, which is based on the principle of the decision-tree and yields to capable sensor selection. Furthermore, the software tool includes an interactive help function, which illustrates the operation instruction of the tool and describes the principles of all available sensors and gives definitions of used technical terms. Basically, both ways should yield to the same result in the proposal of capable sensors for the respective measuring tasks.

\section{DISCUSSION OF STRATEGY CONCEPTS}

The fundamental strategy of measurements with coordinate measuring machines is based on the analysis of single measurement points. These points are used to calculate geometry elements which approximate the inspection feature or serve as foundation for the calculation of the inspection feature.
Dimensional measurements are always associated with fitting algorithms for the appropriate geometry elements. Therefore within the framework of inspection planning the coordinates of touch points are defined. In this connection the number and the position of the touch points are very decisive (radiusmeasurement in Figure 2). This general inspection strategy is well-established. The most producers of coordinate measuring machines offer software applications for the inspection planning. The familiar software applications (Calypso, Quindos) support the import of CAD-data for the definition of measuring points. Using this kind of software, inspection planning can be realised automatically. In principle, this is common practise for tactile- and image sensors and it is used for the most dimensional measurement tasks in the macroscale.

That approved approach can not be translated by implication into the micro- and nanoscale. Even if tactile- and image sensors can be used, CAD-data of the measuring object are not always available or there are excessive deviations between these data and the real unit under test. However, if the topography or also the structures are not known from the available CAD-data, this can turn out to be a real challenge. Here again, image processing may be helpful.

The essential difference is that sensors with other measuring principles are used in the micro- and nanoscale.

In the last few years scanning probe techniques have been rapidly developed. The advantage of scanning sensors is the very high flexibility for adaptation to different scanning areas. These probing systems can acquire measuring data only by scanning the surface sequentially and point-by-point. For measurements on different micro- and nanostructured step height standards uncertainties of less than $1 \mathrm{~nm}$ were reported [2]. The result of measurements with these sensors on smallscale coordinate measuring machines are huge 3D point cloud data. Scanning sensors are e. g. the atomic force microscope (AFM), the white-light interferometer and the laser scanning microscope. Scanning force microscopes are able to measure surfaces with a very high vertical $(<1 \mathrm{~nm})$ and a high lateral resolution $(<10 \mathrm{~nm})$. When inspecting nanostructured features surface metrology and dimensional metrology melt together. Exemplary at AFM measurements the recorded raw measuring data have to be interpreted respectively deconvoluted according to the existing physical as well as geometrical interactions between tip and sample. Otherwise wrong measuring results will be attained [3].

One great challenge of measurements with scanning sensors is to analyse the high volume of measurement data. The second problem is that the strategy used for tactile and image sensors is not capable [8]. Whereas in the macroscale measuring points are defined before the measurement, by using scanning sensors the complete scanning area is measured. The result, a $3 \mathrm{D}$ point cloud has to be analysed (Figure 4c)). Initially it is unknown which points should be used for evaluating the measuring feature. And it would be hard going to select each point based on the image of the point cloud in particular. The available inspection planning software applications do not have the ability to handle such amount of data.

The following approaches are possible ways to analyse the measurement data of scanning sensors.

- Segmentation:

o A segmentation software delivers segmented point clouds with fitted geometry elements. 
o Relevant for the quality of the measurement is to answer the questions: "How good was the fitting process?", respectively "Which points are used for the final fitting??"

- Comparative measurement:

o State of the art is the comparative measurement by insertion of measuring lines in the reconstructed image of the point cloud.

o The disadvantages of this method is that the measuring process can be realised only manually and based on this yields a high measurement uncertainty and there are no performance test results for the determined measurements of geometry elements.

o The advantage is that there are numerous software applications mostly supported by the producers of the sensor.

- Cascaded measurements:

o This method is based on overview images of the measuring scene as alternative to CAD data.

o Local relevant scan areas are selected on the basis of the measuring tasks.

o A complete scan is not necessary. Hence there is a smaller number of measuring points for analysing.

If all these ways will not be successful than functional tests which are usually executed after the assembly of the whole micromechanical product are an alternative way.

The problem of inspection planning is, to solve the special measuring tasks directly connected with the quality of the part to be tested. However, every sensor presents various advantages and disadvantages. The selection of the best probe and the best strategy depends on several parameters of the measuring task [4].

\section{CONCLUSIONS}

There has been a constant improvement of measuring machines and sensors as well as of manufacturing processes. An essential contribution to the improvement in efficiency and usability can be fulfilled by the preparation of knowledge and rules for inspection planning especially for assigning capable sensors to current inspection tasks.

The well known methods and procedures for inspecting macroscopic features respectively the working principles they stand for, was investigated regarding their applicability in inspecting purposeful features at micro- and nanostructured components. Many of the known inspection strategies in dimensional metrology are not likely to be of use under these conditions but some may prove being very useful.

The main result of this paper is the statement that automation of inspection planning yields to objectification of the measurement, reduction of the measurement uncertainty and the measuring time as well as the increase of usability and the reduction of the planning effort.

Topics of further research include methodologies for optimising the measurement strategy and sensor parameter setting using simulations. Thereby the expression of the measurement uncertainty is essential for the inspection planning especially the sensor selection.

\section{ACKNOWLEDGEMENT}

The presented work is the result of the close interdisciplinary research within the SFB 622, which is located at the TU Ilmenau, Germany, and funded by the German Research Foundation (DFG).

\section{REFERENCES}

[1] J. Fleischer, J. Peters, I. Buchholz, "Bewertungssystem zum Vergleich und zur Auswahl von Sensoren in der MultisensorKoordinatenmesstechnik“, Proc. of Mikrosystemtechnik Kongress 2007, Oct. 15-17, 2007, Dresden.

[2] E. Manske, T. Hausotte, R. Mastylo, T. Machleidt, K.-H. Franke, G. Jaeger, "New applications of the nanopositioning and nanomeasuring machine by using advanced tactile and non-tactile probes", Meas. Sci. Technol. 18 (2007) pp. 520-527.

[3] S.C.N. Toepfer, G. Linss, U. Nehse, "Automatic execution of inspection plans for knowledge-based dimensional measurements of micro- and nanostructured components", Proc. of $18^{\text {th }}$ IMEKO World Congress, Sept. 17-22, 2006, Rio de Janeiro, Brazil.

[4] K. Weissensee, "Knowledge-based inspection planning for multiscaled quality testing", Proc. of Joint Int. IMEKO TC1 + TC7 + TC13 Symposium, Aug. 31-Sept. 2, 2011, Jena, Germany.

[5] J. Fleischer, et al. "Erfassung von Standardgeometrieelementen im Mikrometerbereich", Techn. Messen 75 (2008) 5 / DOI 10.1524/teme.2008.0875, Oldenbourg publishing pp. 327-338.

[6] I. Buchholz, "Strategies for quality assurance of micromechanical components with multi-sensor coordinate measurement technique", Shaker, 2008.

[7] A. Burla, T. Haist, W. Lyda, M. H. Aissa, W. Osten, "Assistance systems for efficient multiscale measurement and inspection", Proc. SPIE 8082, 808202 (2011), doi:10.1117/12.889344.

[8] E. Manske, G. Jaeger, T. Hausotte, "Combination of multisensor technology and multiple measurement strategies in microand nanometrology", Proc. of ISMQC-2010 10th International Symposium on Measurement an Quality Control, Sept. 5-9, 2010, Osaka, Japan. 